\title{
A comparison between radiochromic EBT2 film model and its predecessor EBT film model
}

\author{
M.A. Carrasco*, M. Perucha, F.J. Luis, M. Baeza, M. Herrador
}

Department of Medical Physics, Virgen del Rocio Hospital (Seville), Sevilla, Spain

Received 13 January 2012; received in revised form 17 May 2012; accepted 21 May 2012 Available online 26 June 2012

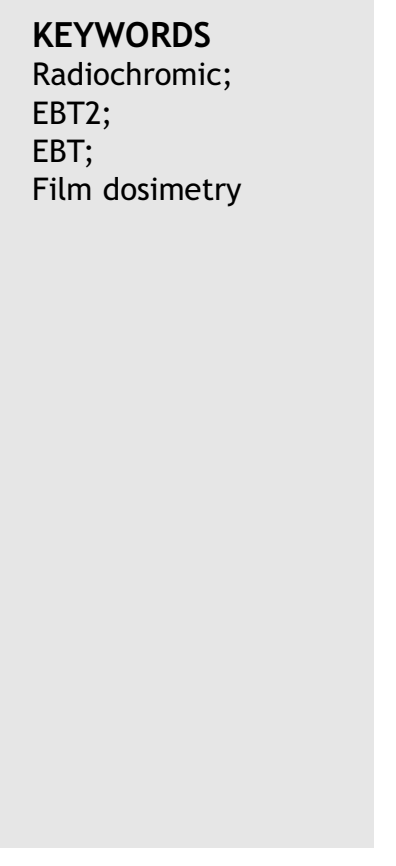

\begin{abstract}
The manufacturer has introduced the new EBT2 film model so as to improve its predecessor, the EBT radiochromic film model. According to the manufacturer, some of its main advantages include a higher tolerance to light exposure and it can correct nonuniformity of the active layer thickness using a marker dye. However, the equivalence in uniformity between both models was questioned by some authors, and the asymmetrical configuration of layers of the EBT2 film model produces a new dependence on the film side being scanned (front and back orientation). In this study, the EBT2 radiochromic film model was compared with the EBT model and the new marker dye feature was assessed. We also compared this correction method with a pre-irradiated pixel value correction method. An Epson Expression 10000XL scanner in transmission mode was used to scan the films and the red channel response was analyzed. We confirmed the lower-measured signal dependence on the visible light exposure of the EBT2 film model. Differences in pixel values remained below $0.5 \%$ for a minimum of 15 days. In regard to the uniformity, similar results for EBT2 and EBT film models were obtained; in both cases inhomogeneity was found to be less than $1 \%$, in relative pixel value from the mean. However, we found that the signal-to-noise ratio was reduced for low doses by $37 \%$ for old EBT2 batch and by $21 \%$ for new EBT2 batch compared to signal-tonoise ratio for EBT. The EBT2 film model's pixel value difference for the front and back orientation reached up to $1.0 \%$ in the red channel. Our results did not show a clear advantage between to use a pre-irradiated pixel value correction and to use the manufacturer's correction.

(c) 2012 Associazione Italiana di Fisica Medica. Published by Elsevier Ltd. All rights reserved.
\end{abstract}

\footnotetext{
${ }^{*}$ Corresponding author.

E-mail address: macarrasco_her@yahoo.es (M.A. Carrasco).
}

1120-1797/\$ - see front matter ( $) 2012$ Associazione Italiana di Fisica Medica. Published by Elsevier Ltd. All rights reserved. doi:10.1016/j.ejmp.2012.05.008 


\section{Introduction}

Radiographic film was the most commonly used verification system in radiotherapy for years. Nowadays, new radiochromic films are increasingly used due to their greater sensitivity and their wider useful dose range in comparison to traditional films. Moreover, as radiochromic film is selfdeveloping, no chemical processing is needed, and it can be handled and prepared in room light. Therefore, it has been established as a verification system for 3DCRT treatments [1-3], IMRT [4-7], dynamic arc therapy and brachytherapy $[8,9]$. In addition, radiochromic films are useful for quality control of linear accelerators [10,11].

The Gafchromic ${ }^{\circledR}$ EBT was the first model to be widely used for dosimetry (International Specialty Products, ISP) [12]. The main features of these films were: sensitivity range between $1 \mathrm{cGy}$ and $800 \mathrm{cGy}$; energy independence between $\mathrm{keV}$ and $\mathrm{MeV}$; nearly tissue equivalent $\left(z_{\text {eff }}=6.98\right)$; they can be immersed in water for short periods of time, and they can be cut into several pieces.

These films have been analyzed and characterized in many papers [13-17]. According to these studies, the EBT model was reliable for dosimetry, although a film dosimetry protocol was required to minimize the dosimetric uncertainties. The most important factors are the landscapeportrait orientation of the film on the scanner, inhomogeneity of the scanner response, film inhomogeneity, and the time delay between irradiation and scanning.

This film model has been replaced by the Gafchromic ${ }^{\circledR}$ EBT2 model [18], which is different to the previous model its yellow color being the most evident difference. This arises from the presence of a yellow dye incorporated into the active layer, whose purpose is to improve the homogeneity by using the response of the yellow dye in the blue channel. Moreover, the active layer is sensitive to blue light, which the yellow dye absorbs, thereby reducing the light dependence of the radiochromic films. The manufacturer has cited other improvements, i.e. less energydependent, because a natural polymer has been replaced by a synthetic polymer. This has improved the safety of the film's composition, and reduced damage to the film when cut due to the use of an over-laminate with pressure sensitive adhesive.

Gafchromic EBT2 films' energy dependence has been extensively studied [19-22]. However, to date there are few studies about other radiochromic EBT2 films' characteristics [23-26], the film uniformity being the most controversial issue $[27,28]$. The lack of uniformity has several contributions [29], on the one hand, Newton's ringlike scanning artifacts, which can be avoided by elevating the film above the scanner's surface, on the other hand, the inhomogeneity of the film itself, which depends on the coating technology. The manufacturer proposes a correction procedure to improve this second kind of inhomogeneity using the marker dye's blue absorption band. Only one study [29] takes the use of this procedure into account, resulting in disagreement with the manufacturer, since the uniformity is worse when the manufacturer's recommended procedure is used. The film uniformity of some early batches investigated [28] shows unacceptable values for clinical quality assurance. A review has been recently published [30] that urges homogeneity and correction procedure research to continue, while the film manufacturing becomes stable. Given the previous results, we investigated the uniformity in this study, including the manufacturer's recommended procedure, on batches of EBT2 films. Besides, we studied other characteristics of these films using the Epson Expression 10000XL scanner and we have compared them with the results obtained for EBT films. A recent study [31], assessed some EBT2 model characteristics comparing them with the EBT film, however, there are important items like film and scanner uniformity that were not studied. Centers have film dosimetry protocols established for the EBT model, meaning that the differences and similarities with the EBT2 model must be studied so that necessary changes in these protocols can be made. The factors we have considered are: light sensitivity, orientation of the film, reproducibility with the number of previous scans, position of the film in the scanner, time between irradiation and scanning, and film uniformity.

\section{Materials and methods}

In this work we characterized the EBT2 Gafchromic ${ }^{\circledR}$ films, digitized with an Epson Expression 10000XL scanner, which allows A3 scan sizes. In our protocol, all the films were stored at room temperature in an opaque envelope in order to protect them from visible light. They were cut into $4 \times 5 \mathrm{~cm}^{2}$ pieces on the day of irradiation, marked in the upper right corner, and scanned individually before irradiation and after $24 \mathrm{~h}$. For studies using the EBT film model we have used one batch, 47277-04l. For studies using the EBT2 model we have used films from the F031810001A batch, but for the homogeneity study we used two batches, F10070902A and A09171002. The films used for this study were irradiated under calibration conditions: we used a $6 \mathrm{MV}$ photon beam for irradiation (Varian 600C LINAC, Varian Medical Systems, Palo Alto, CA) a solid water phantom, at a depth of $1.5 \mathrm{~cm}$, and with at least $10 \mathrm{~cm}$ water phantom behind it to provide full backscatter conditions. The beam field size was $10 \times 10 \mathrm{~cm}^{2}$ at the isocenter and the source-surface distance was $100 \mathrm{~cm}$.

The Epson Expression 10000XL scanner is recommended by the EBT2 film manufacturer. Since this scanner is A3 in size, opaque black cardboard frames should be placed in the scanner to ensure that the film is positioned in the center, without having to remove it during scanning $[15,16]$. We used the Epson Scan software, in the professional mode. Settings were created to always keep the same regions of interest (ROI). A preview image was acquired and afterwards the film was scanned in 'positive' mode, which produces low pixel values in dark areas, without color correction, with resolution of $72 \mathrm{dpi}$, using transmission mode and 48 bit depth [14]. The image was exported in TIFF format and analyzed by ImageJ (ImageJ software 2010) [32], choosing the red channel for the dosimetry because the active layer's maximum absorption is $636 \mathrm{~nm}$ [33], showing more sensitivity in the range of doses commonly used in the clinical setting [1]. We used a $1.4 \times 1.4 \mathrm{~cm}^{2}$ scan area to study the films [14] and we used an ImageJ's macro to ensure the reproducibility of its position. Film darkening was quantified through the pixel 
value and the equivalent dose. The equivalent dose was obtained from a calibration curve for each batch, irradiating from $5 \mathrm{cGy}$ to $405 \mathrm{cGy}$, obtaining 24 points besides the point corresponding to the non-irradiated film. The points were fitted to a third order polynomial [34]:

Dose $=a \mathrm{OD}^{3}+b \mathrm{OD}^{2}+c \mathrm{OD}+d$

In order to apply the homogeneity correction proposed by the manufacturer we used the optical density (OD) [34], instead of net optical density [13], calculated from the pixel value (PV) applying:

$O D=\log _{10}\left(\frac{65535}{P V}\right)$

where 65,535 is the maximum pixel value for a 16 bit channel.

The dose uncertainties were calculated by error propagation as proposed by Devic [35]. We considered two sources of uncertainties: experimental and fitting. Experimental uncertainties were associated with the film irradiation and scanning. Following Devic [35], we only took into account the reproducibility in measuring the OD as a contributor to the experimental uncertainty. The optical density uncertainty was calculated using:

$\sigma_{\mathrm{OD}}=\frac{1}{\ln 10} \frac{\sigma_{\mathrm{PV}}}{\mathrm{PV}}$

Where $\sigma_{\mathrm{PV}}$ is the standard deviation obtained from the pixel values in the region of interest.

The final experimental dose uncertainty was calculated applying:

$\sigma_{\mathrm{e}-\mathrm{dose}}=\left(3 a \mathrm{OD}^{2}+2 b \mathrm{OD}+c\right) \sigma_{\mathrm{OD}}$

Fitting uncertainties were associated with the accuracy of the fitting process. The standard deviation of the fitting parameters was calculated using the fitting software Table Curve 2D ${ }^{\circledR} 5.01$ (Systat Software Inc, London, UK).

$\sigma_{\mathrm{f}-\text { dose }}=\sqrt{\mathrm{OD}^{6} \sigma_{a}^{2}+\mathrm{OD}^{4} \sigma_{b}^{2}+\mathrm{OD}^{2} \sigma_{c}^{2}+\sigma_{d}^{2}}$

Finally, the total dose uncertainty was calculated using the expression:

$\sigma_{\text {dose }}=\sqrt{\sigma_{\mathrm{e}-\text { dose }}^{2}+\sigma_{\mathrm{f}-\text { dose }}^{2}}$

We analyzed the influence of the following parameters in the pixel value and dose:

\section{Light sensitivity}

Radiochromic films, particularly EBT films, are sensitive to blue wavelengths [36]. The manufacturer states that the EBT2 film is less sensitive to indoor light than the EBT, because the yellow dye added to the active layer strongly absorbs blue light. The EBT2 film's light sensitivity has been previously studied by Richley et al. [27] and Andrés et al. [31]. Richley et al. [27] studied the effect of light on film and investigated the film's response to daylight and to fluorescent strip lighting. However, their findings are not comparable with the rest of the literature because they did not measure the light intensity with a lux meter, and their results were reported in ADC values. Andrés et al. [31] studied EBT2 films under high exposure to indoor light (1600 lux). They found a darkening equivalent to a dose of $6 \mathrm{cGy}$ for $2 \mathrm{~h}$ of exposure, and $20 \mathrm{cGy}$ for $6 \mathrm{~h}$ of exposure. According to their results, working with radiochromic EBT2 films under high exposure to indoor light should be highly minimized in time and light exposure. To study the darkening that occurs in relatively short times of exposure under normal operating conditions, we kept a piece of EBT2 film in a room with fluorescent bulbs under normal indoor illuminations (500 lux) and it was scanned in short intervals during $4 \mathrm{~h}$.

On the other hand, according to the manufacturer, EBT2 film model is not totally insensitive to light. To quantify this fact, we studied the light sensitivity of EBT and EBT2 model under low exposure to indoor light. The light intensity was 150 lux, measured with the lux meter Nuclear Associates 07-621 Precision Photometer (Quantum instruments INC). We kept pieces of both EBT2 and EBT non-irradiated films in a room with windows and fluorescent bulbs, without exposing them to direct light for 15 days. They were scanned every day to check how light exposure could affect the darkening.

\section{Front and back orientation of the film}

Unlike the EBT model, the configuration of the layers of the EBT2 film is not symmetrical. The effect from scanning the film on the 'front' side versus the 'back' side was analyzed by Desroches et al. [25] in terms of net optical density. They concluded that the net optical density difference given by scanning on alternate sides is significant only in reflection scanning mode but not in transmission mode. Moreover, Aldelaijan et al. [23], concluded that the difference is not significant, but they did not quantify it. On the other hand, the manufacturer recommends always scanning the same side of the film facing the scanner lamp due to a potential scanner variation response of several per cent; however, he does not quantify this variation. To analyze this effect, we scanned both sides of the $4 \times 5 \mathrm{~cm}^{2}$ pieces of both EBT and EBT2 films, non-irradiated and irradiated with 20,100 and 200 cGy.

\section{Landscape-portrait orientation of the film}

The flatbed scanners outcome depends on the 'landscape' or 'portrait' orientation of the film on the scanner: 'landscape', when the short side of the film is parallel to the long side of the scanner, and 'portrait', when it is perpendicular. This is due to the fact that the needle-shaped composition of the active layer produces anisotropic light scattering. Martisikova et al. [14] evaluated the errors coming from rotating EBT films as a function of dose. To evaluate this effect, we performed irradiations from 0 to $225 \mathrm{cGy}$ on $4 \times 5 \mathrm{~cm}^{2}$ pieces of both EBT and EBT2 films and we analyzed the differences between the two orientations.

\section{Reproducibility with the number of scans}

To study the reproducibility with the number of scans we have considered two possible effects: scanner warming and film darkening with the number of scans. 
Some studies $[14,16,27,34]$ recommend not using the first scans because of a possible scanner warming effect. Martisikova et al. [14] and Ferreira et al. [16] reported a warming up effect of the scanner lamp with the Epson Expression 10000XL scanner. Richley et al. [27] observed a maximum difference in signal of $0.2 \%$ with the same scanner, with no trend occurring over consecutive scans. We have studied this effect with two types of film, scanning the same film from 0 (preview) up to 10 times without waiting at any time between scans.

These studies also describe an influence of the scanner lamp on films due to multiple scans. For EBT film model, Paelinck et al. [34], with the scanner Epson 1680 pro, and subsequently Martisikova et al. [14], with the scanner Epson Expression 10000XL, found a darkening of the film due to multiple scans. For the EBT2 film model, Richley et al. [27], did not find an effect of repeated scanning on film; they suggest that EBT2 film is insensible to the ultraviolet light emitted by the scanning light and any increase in temperature of the scan glass. We have studied this effect with both types of film by scanning the same film from 0 (preview) to 50 times every $5 \mathrm{~min}$. Both tests were made for doses of 20, 100 and $200 \mathrm{cGy}$.

\section{The position of the film in the scanner}

We have analyzed the dependence of the pixel value on the film position in the scanner, performing scans in the center and 8 other positions (Fig. 1) which result from combinations between $Y$ direction (up, center, down) and $X$ direction (left, center, right). Some studies have reported [14-16] that the scanner has an inhomogeneity response in the direction perpendicular to the movement $(\mathrm{Y})$ caused by light scattering in the scanner. This effect depends on the dose delivered to the film; therefore irradiated films with 20, 100 and 200 cGy have been analyzed. We always scanned the same piece of film $\left(4 \times 5 \mathrm{~cm}^{2}\right)$ to separate this effect from the inhomogeneity of the film itself. A black cardboard frame (A3 size) with nine holes of $4 \times 5 \mathrm{~cm}^{2}$ size corresponding to the different positions was used to control them.

\section{Time between irradiation and scanning}

The optical density of Gafchromic film increases with time. The darkening of films irradiated with lower doses stabilizes

\begin{tabular}{|c|c|c|}
\hline $\mathbf{1}$ & $\mathbf{2}$ & $\mathbf{3}$ \\
$(-10.5 \mathrm{~cm}, 8.5 \mathrm{~cm})$ & $(0 \mathrm{~cm}, 8.5 \mathrm{~cm})$ & $(10.5 \mathrm{~cm}, 8.5 \mathrm{~cm})$ \\
\hline $\mathbf{4}$ & $\mathbf{5}$ & $\mathbf{6}$ \\
$(-10.5 \mathrm{~cm}, 0 \mathrm{~cm})$ & $(0 \mathrm{~cm}, 0 \mathrm{~cm})$ & $(10.5 \mathrm{~cm}, 0 \mathrm{~cm})$ \\
\hline $\mathbf{7}$ & $\mathbf{8}$ & $\mathbf{9}$ \\
$(-10.5 \mathrm{~cm},-8.5 \mathrm{~cm})$ & $(0 \mathrm{~cm},-8.5 \mathrm{~cm})$ & $(10.5 \mathrm{~cm},-8.5 \mathrm{~cm})$ \\
\hline
\end{tabular}

Figure 1 Diagram of the positions where the pieces of film have been scanned. earlier than that of films irradiated with higher doses $[15,18,37]$. For the EBT model, the standard was to wait $24 \mathrm{~h}$ after irradiation to scan, although published studies establish a minimum delay of $6 \mathrm{~h}$ [37]. For the EBT2 model, the effect of darkening over time has been studied previously [27,31] for short intervals within the first $24 \mathrm{~h}$. Our intention was to study how film darkening varies for longer periods of time, to consider possible situations encountered in practice, for example, delay times during weekends. We irradiated a piece of film with $100 \mathrm{cGy}$ and scanned it every hour for $72 \mathrm{~h}$. The test was performed with two pieces of the same film sheet delaying irradiation by $12 \mathrm{~h}$ to cover the first $24 \mathrm{~h}$. We compared the results for the EBT2 film with those obtained with EBT films. For the EBT2 model this item was deeply investigated. We irradiated a piece of film with $100 \mathrm{cGy}$ and scanned it with a reference color scale tablet present in the scanning region to correct the pixel values for variations due to scanner repeatability. We scanned it every hour during the first twelve hours and afterwards every six hours finishing $72 \mathrm{~h}$ from irradiation.

\section{Film uniformity}

Homogeneity of the EBT film has been analyzed previously by other authors; Fuss et al. [15] studied it in detail, including sheet to sheet homogeneity, intrasheet homogeneity and profiles. There is agreement between the homogeneity obtained in this study and other studies $[14,16]$. However, the results for the EBT2 film homogeneity are not consistent, and strongly depend on the batch used. In the first published works, the homogeneity of the EBT2 film was not acceptable for clinical use [28], although a subsequent study with a different batch, shows better homogeneity results [27]. To analyze the possible influence of the batch in the homogeneity of the film we used EBT2 films from an older and a more recent batch (F031810001A and A09171002, respectively). We have evaluated the sheet to sheet homogeneity and the intrasheet homogeneity for both film models. In addition, we analyzed the signal-to-noise ratio of both models. In order to study which are the consequences of this lack of homogeneity on dose, we exposed some films from different models and batches to a homogeneous field. Finally, we studied the profiles and we have applied the correction protocol proposed by the manufacturer to improve the homogeneity for EBT2.

The procedure to evaluate these five items is as follows:

1. Sheet to sheet homogeneity. To do this, the pixel value was measured in a central ROI of 10 sheets of the same non-irradiated batch.

2. To evaluate the intrasheet homogeneity and its dependence on the dose, three groups of ten pieces $4 \times 5 \mathrm{~cm}^{2}$ of the same sheet were cut and irradiated with 20, 100 and $200 \mathrm{cGy}$, respectively, and the pixel value was measured in a central ROI.

3. The signal-to-noise ratio (SNR) was evaluated by the ratio of the average pixel value in a ROI of $1.4 \times 1.4 \mathrm{~cm}^{2}$ $\left(\mathrm{PV}_{\text {mean }}\right)$ and the standard deviation $(\sigma)$ for pieces of nonirradiated and irradiated films with 20, 100 and 200 cGy.

4. We analyzed the central part of three horizontal profiles corresponding to a film of the three batches (EBT, EBT2 F031810001A, EBT2 A09171002) irradiated with $10 \times 10 \mathrm{~cm}^{2}$ uniform fields at $100 \mathrm{cGy}$. 
5. We analyzed horizontal profiles of two $4 \times 5 \mathrm{~cm}^{2}$ films irradiated with $10 \times 10 \mathrm{~cm}^{2}$ uniform fields at 100 and $200 \mathrm{cGy}$, and one non-irradiated film, to assess the uniformity correction protocol proposed by the manufacturer [18]. It includes the blue channel response, which depends on variations in the thickness of the active layer of the film. The correction protocol had four steps:

a) For each of the 24 pieces of calibration films we obtained the red optical density $\left(O D_{\text {red }}\right)$ and the blue optical density $\left(\mathrm{OD}_{\text {blue }}\right)$ by means of eq. (2).

b) We plotted the red optical density versus the ratio red:blue optical density $\left(O D_{\text {red }} / O D_{\text {blue }}\right)$, and we fitted it to a second order polynomial.

c) We applied the above function to convert the red:blue optical density into a corrected value of the optical density in the red channel (corrected $\mathrm{OD}_{\text {red }}$ )

d) We plotted the corrected $O D_{\text {red }}$ versus the applied dose to obtain a corrected calibration curve and we fitted it to a third order polynomial.

Finally, we used the above functions to convert the red:blue optical density of each pixel of five horizontal profile into dose. We compared the standard deviation of the profile using the correction procedure and without using it. We also studied profiles obtained using net optical density from the pre-irradiated pixel value [29].

\section{Results and discussion}

\section{Light sensitivity}

Under normal exposure to indoor light our results do not show a significant dependence on light exposure of the EBT2 films (Fig. 2) when we exposed the film for $4 \mathrm{~h}$ continuously. We found a maximum difference of $0.5 \%$ in pixel value for the red channel (equivalent to a dose of $0.2 \pm 1.8 \mathrm{cGy})$.

Under low exposure to indoor light our results show a significant decrease in the dependence on light exposure of the EBT2 films compared to the EBT (Fig. 3). In the case of EBT films we found a difference of $0.8 \%$ in pixel value for the red channel (equivalent to an absolute dose difference with respect to the initial dose of $2.3 \pm 1.3 \mathrm{cGy}$ ) at $48 \mathrm{~h}$, reaching a difference of $4.3 \%(11.4 \pm 1.4 \mathrm{cGy})$ at 15 days. However, for the EBT2 films the differences remained below $0.5 \%$ in pixel value $(0.2 \pm 1.8 \mathrm{cGy})$ for a minimum of 15 days.

According to the results found by other authors, sensitivity to light is highly dependent on the intensity with which they are exposed. Andrés et al. [31] found that the EBT2 films were darker than in this study when exposed to higher light intensities (Andrés 1600 lux). In conclusion, we can accurately work with the EBT2 films exposed to standard indoor light, as long as we take care to avoid unnecessary light exposure.

\section{Front and back orientation of the film}

No differences in pixel value occur for the EBT film when scanning with different orientations (Fig. 4), being less than $0.3 \%$ for the red channel (equivalent to an absolute dose

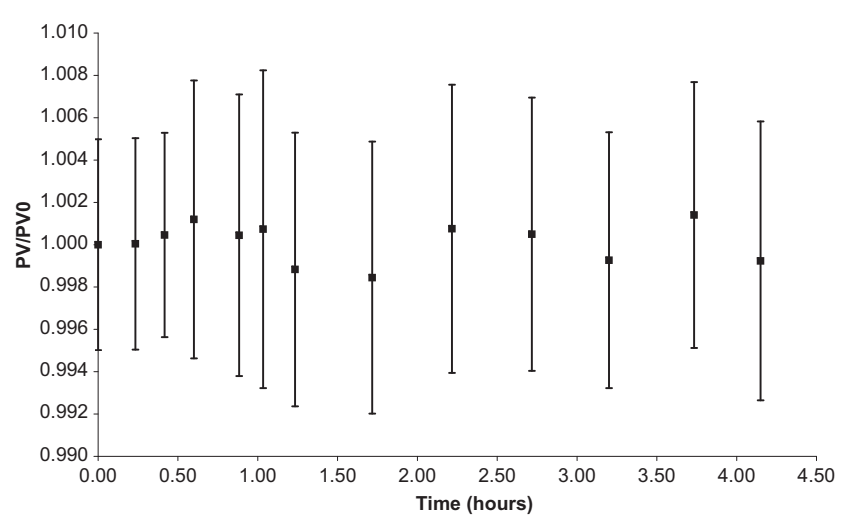

Figure 2 Ratio between $\mathrm{PV}$ and pixel value from the first scan $\left(\mathrm{PV}_{\mathrm{o}}\right)$ for EBT2 film exposed to standard lighting. Continuous exposure during $4 \mathrm{~h}$ does not darken the film significantly. The error bars correspond to one standard deviation calculated by error propagation from the expression $P V / P V_{0}$.

difference of $0.8 \pm 0.9 \mathrm{cGy})$. For the EBT2 film, the differences can reach $1.0 \%$ for 200 cGy $(7.0 \pm 5.0$ cGy).

These results correspond to the difference in net Optical Density obtained by Desroches et al. [25] for EBT2. Although ours results agree with Desroches's quantitative measurements, we would like to emphasize that the difference found is significant and it can reach $1 \%$. To avoid this uncertainty, the manufacturer recommends scan always the same side of the film in order to minimize dosimetric uncertainties.

\section{Landscape-portrait film orientation}

We found large discrepancies between the landscape and portrait measurements in the EBT2 film, we obtained relative differences in pixel value from $3.6 \%$ (equivalent to an absolute dose difference of $15.9 \pm 1.5 \mathrm{cGy}$ ) to $6.8 \%$ (53.5 $\pm 5.9 \mathrm{cGy}$ ) depending on the applied dose, which increased as it did. The results for the EBT film are in the range from $4.9 \%(13.2 \pm 0.9 \mathrm{cGy})$ to $6.0 \%$ (35.8 $\pm 4.4 \mathrm{cGy})$.

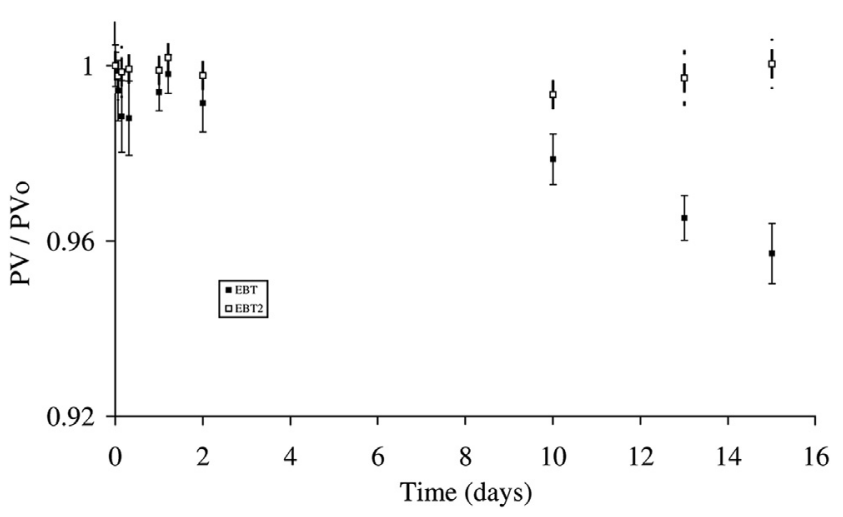

Figure 3 Ratio between PV and pixel value from the first scan $\left(\mathrm{PV}_{\mathrm{o}}\right)$ for EBT film (solid square) and EBT2 film (empty square) exposed to low lighting. EBT2 film has no appreciable darkening due to light exposure for two weeks. The error bars correspond to one standard deviation calculated by error propagation from the expression $\mathrm{PV} / \mathrm{PV}_{\mathrm{o}}$. 


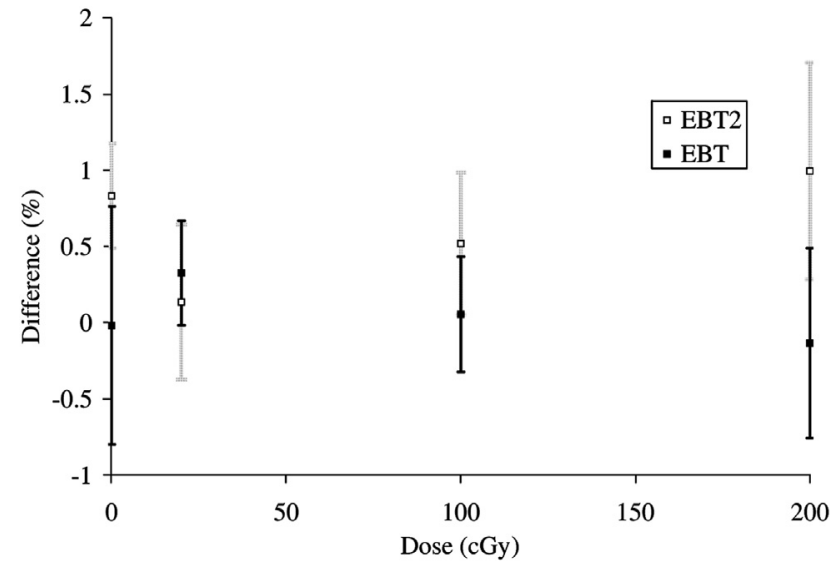

Figure 4 Relative difference between front and back orientations for EBT film (solid square) and EBT2 film (empty square). EBT2 film shows a slight dependence on front and back orientation. The error bars correspond to one standard deviation calculated by error propagation from the expression $\left(P V_{\text {from }}-P V_{\text {back }}\right)^{*} 100 / P V_{\text {back }}$.

It is therefore critical to choose one orientation as a reference.

Our results confirm those obtained by other authors for the EBT film [14] and those obtained in recent studies on the EBT2 film [31], finding no difference between these two models for this dependence.

\section{Reproducibility with the number of previous scans}

When analyzing the possible warming effect of the scanner, the observed differences in pixel values of the red channel for both the EBT and EBT2 film remain below $0.1 \%$ (equivalent to an absolute dose difference with respect to initial dose of $1 \pm 1 \mathrm{cGy}$ ), therefore there is no such effect.

Darkening is not observed with the number of scans; the differences in the pixel value was about $0.5 \%$ (equivalent to a dose of $1.9 \pm 1.9 \mathrm{cGy}$ ) between the first and last scan for EBT film (Fig. 5a). We obtained the same outcome for the EBT2 film (Fig. 5 b); differences were always lower than $0.5 \%(2.0 \pm 2.0 \mathrm{cGy})$.

Our results confirm a recent study on EBT2 [27] which found no effect of warming or darkening. The authors attribute this result to the scanner's lower sensitivity to UV light for the EBT2 model. However, our results do not show a warm up effect or a darkening due to multiple scans, for a

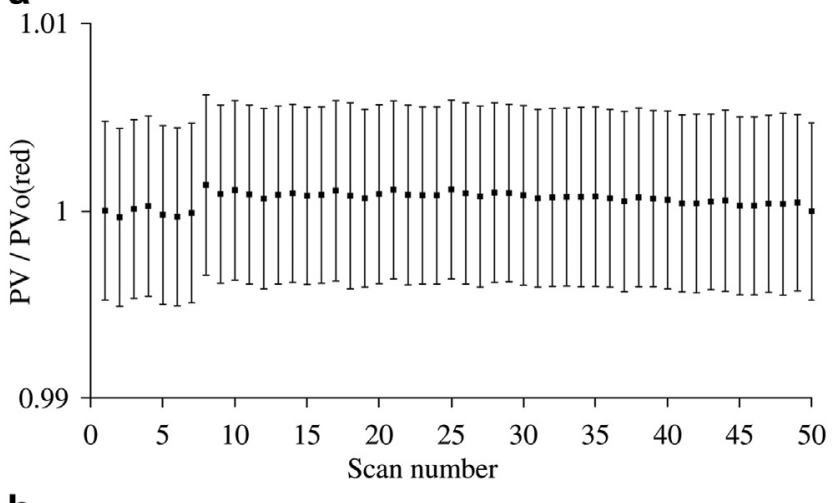

b

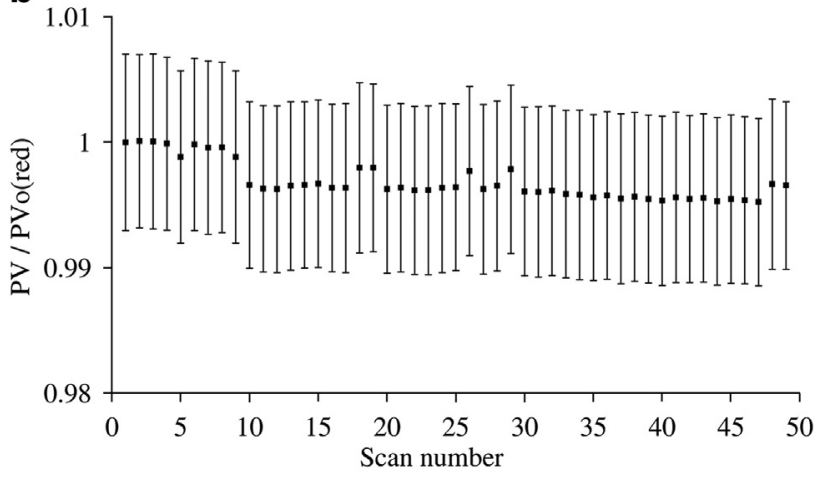

Figure 5 Ratio between PV and pixel value from the first scan $\left(\mathrm{PV}_{\mathrm{o}}\right)$, obtained from scanning a piece of film irradiated with 20 cGy. Fig. 5 a shows the EBT film, Fig. 5 b shows the EBT2 film. The error bars correspond to one standard deviation calculated by error propagation from the expression $P V / P V_{0}$.

neither EBT nor EBT2 film model. Consequently, there is no difference in this feature between both models.

\section{The position of the film in the scanner}

There is a maximum difference of $1 \%$ in pixel value for EBT2 films between each of the tested positions in the horizontal direction of the scanner, and there is no significant dependence on the amount of radiation to which the film is exposed. However, the differences are much greater in the vertical direction and it depends on the applied dose to the film (Fig. 6). For low doses (20 cGy) the maximum pixel value deviation from central position is $1.8 \%$ (corresponding to equivalent to an absolute dose difference with respect
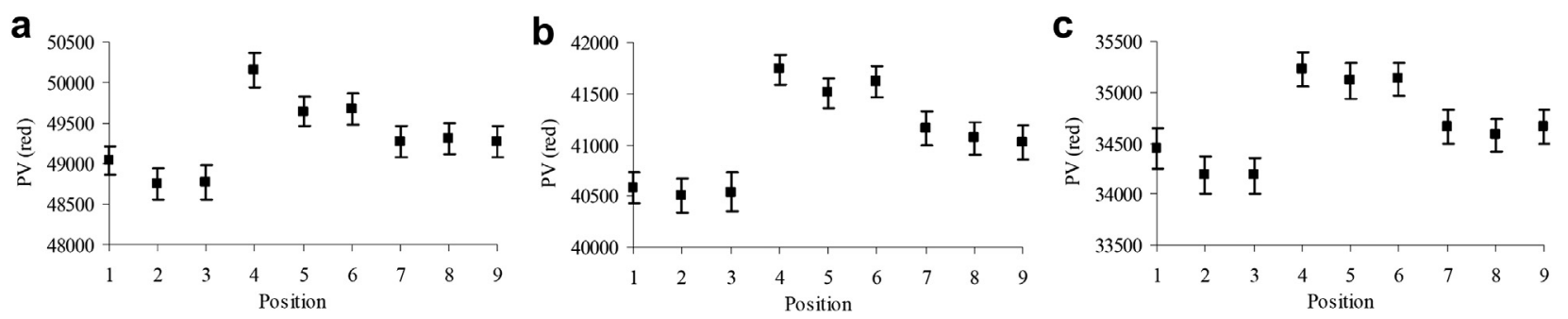

Figure 6 Influence of scanning the pieces of EBT2 films irradiated with a) 20 cGy, b) 100 cGy and c) 200 cGy in different positions specified in Fig. 1. The error bars correspond to one standard deviation $\left(\sigma_{\mathrm{PV}}\right)$. 

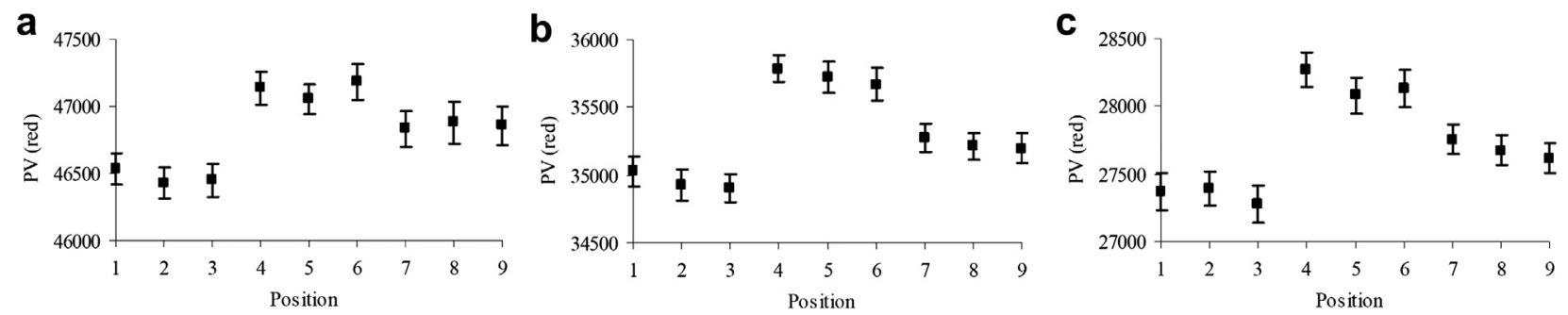

Figure 7 Influence of scanning the pieces of EBT films irradiated with a) 20 cGy, b) 100 cGy and c) 200 cGy in different positions specified in Fig. 1. The error bars correspond to one standard deviation $\left(\sigma_{\mathrm{PV}}\right)$.

to central dose of $7.7 \pm 2.3 \mathrm{cGy})$, increasing as the dose rises, reaching up to $2.6 \%$ (18.9 $\pm 5.1 \mathrm{cGy})$ for high doses (200 cGy).

In the predecessor EBT film (Fig. 7) the differences were smaller in the longitudinal direction of the scanner, with a maximum of $0.6 \%$ in pixel value; both EBT and EBT2 films have the same behavior with the vertical direction dose. The maximum deviation from the central position for EBT ranges from $1.3 \%$ (3.4 $\pm 0.9 \mathrm{cGy}$ ) for low doses (20 cGy) to $2.9 \%(16.1 \pm 3.7 \mathrm{cGy})$ for high doses (200 cGy). Our results show no significant differences between the two models.

The results for the EBT model are within the range of those published by other authors for other scanners $[1,34,38]$, and for the Epson Expression 10000XL scanner [16]. This effect has also been studied by Richley et al. [27] for the EBT2 film but the results are not comparable because they are in terms of analog digital conversion (ADC).

Special care must be taken when analyzing large areas of film; a correction factor should be applied $[1,39,40]$ in order to minimize these differences.

\section{Time between irradiation and scanning}

For the EBT2 model the pixel values corrected for the reference color scale tablet (Fig. 8) show that it darkens strongly during the first hours after irradiation. Seven hours later, its pixel value deviation was reduced to $0.3 \%$ from the

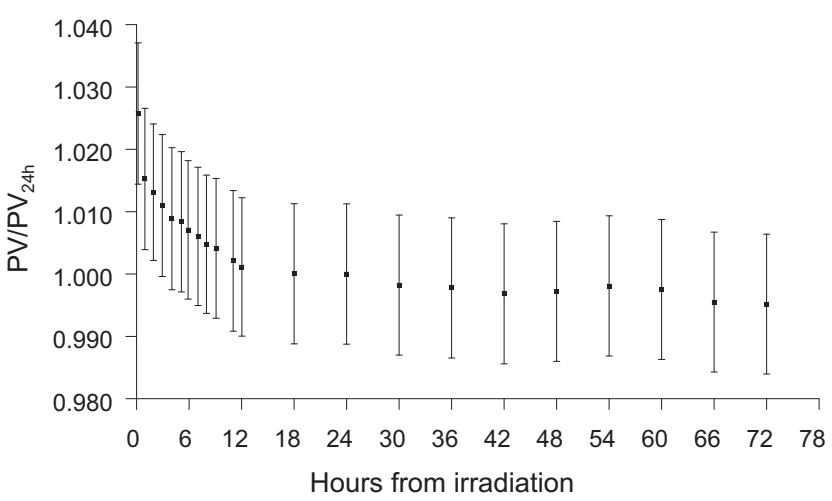

Figure 8 Relative difference between PV post-irradiation and $\mathrm{PV}$ at $24 \mathrm{~h}$ for a piece with a dose of $100 \mathrm{cGy}$ for EBT2 film using a reference color scale tablet. The error bars correspond to one standard deviation calculated by error propagation from the expression $\mathrm{PV} / \mathrm{PV}_{24 \mathrm{~h}}$. film at $24 \mathrm{~h}$ post-irradiation (equivalent to a dose at $24 \mathrm{~h}$ of $1.4 \pm 1.8 \mathrm{cGy}$ ). These deviations remained around $0.5 \% 72 \mathrm{~h}$ after irradiation; indicating that films can be scanned accurately after a weekend.

Some authors [31] state that the EBT2 model is stabilized earlier than the EBT model. In our study, the EBT2 film tends to stabilize rapidly, but we did not find any significant differences, apart from the uncertainty associated with the measure.
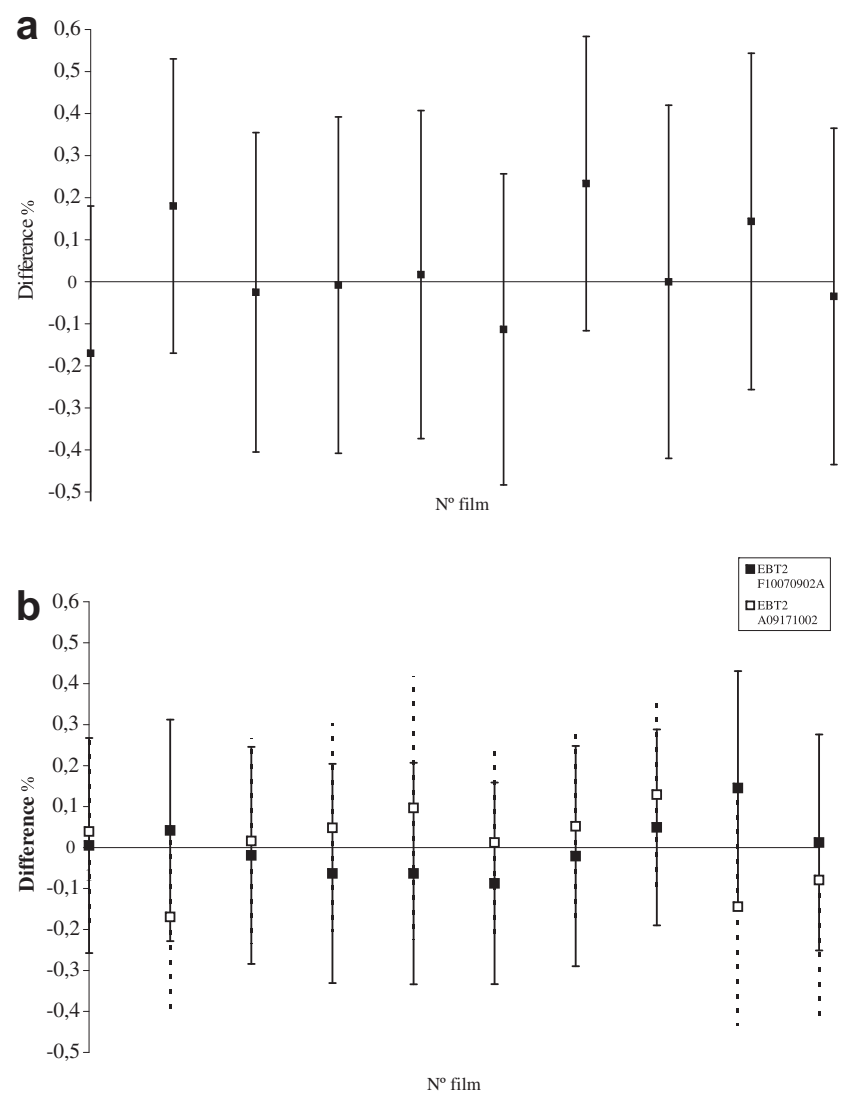

Figure 9 Relative difference of the mean PV resulting from scanning 10 non-irradiated films belonging to the same batch: a) EBT b) EBT2 new batch (solid square), old batch (empty square). The error bars correspond to one standard deviation calculated by error propagation from the expression $\left(P V-P V_{\text {mean }}\right)^{*} 100 / \mathrm{PV}_{\text {mean }}$. 
Table 1 Percentage of the pixel value deviation from the mean, PV (\%), and equivalent dose for non-irradiated films of the same batch, and films of the same sheet irradiated with $20 \mathrm{cGy}, 100 \mathrm{cGy}$ and $200 \mathrm{cGy}$.

\begin{tabular}{|c|c|c|c|c|c|}
\hline & & \multicolumn{2}{|c|}{ EBT2 F031810001A } & \multicolumn{2}{|c|}{ EBT2 A09171002 } \\
\hline & & PV (\%) & Dose (cGy) & PV (\%) & Dose (cGy) \\
\hline Intersheet & Non-irradiated & $-0.2,0.1$ & $1 \pm 1$ & $-0.1,0.1$ & $1 \pm 1$ \\
\hline \multirow[t]{3}{*}{ Intrasheet } & 20 cGy & $-0.2,0.2$ & $1 \pm 1.3$ & $-0.4,0.4$ & $3 \pm 1.3$ \\
\hline & 100 cGy & $-0.4,0.6$ & $3.1 \pm 2.3$ & $-0.3,0.4$ & $3.5 \pm 2.6$ \\
\hline & 200 cGy & $-0.4,0.8$ & $5.2 \pm 4.1$ & $-0.6,0.5$ & $7.3 \pm 3.3$ \\
\hline
\end{tabular}

\section{Film uniformity}

1. For the EBT batch analyzed, the homogeneity between non-irradiated films of the same batch as a percentage of the pixel value deviation from the mean is $[-0.2 \%]$ to [0.2\%] equivalent to an absolute dose difference with respect to mean dose of $1 \pm 1 \mathrm{cGy}$ (Fig. 9a). Table 1 shows the intersheet homogeneity results as a percentage of the pixel value deviation from the mean for the EBT2 batch (F031810001A) and the recent EBT2 batch (A09171002) (Fig. 9 b). There are no noticeable differences in the intersheet homogeneity between the EBT2 batches studied, or between them and the EBT model batch.

2. We found a slight dependence on the dose when analyzing the homogeneity of the pieces cut from the

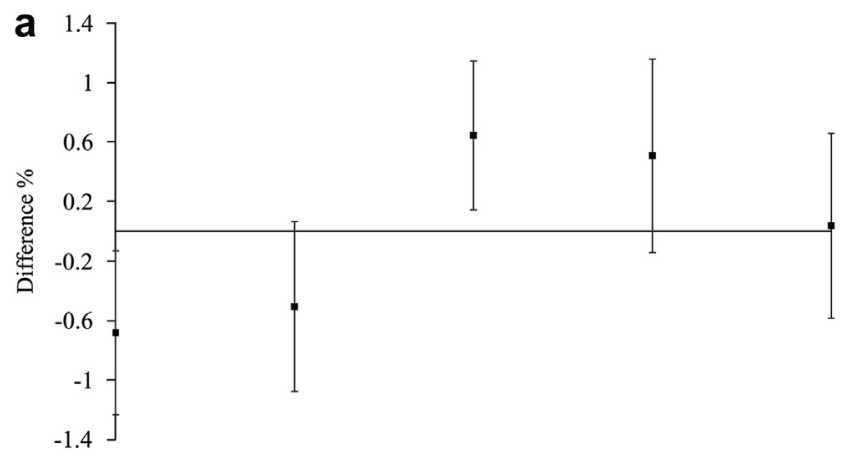

Piece of film

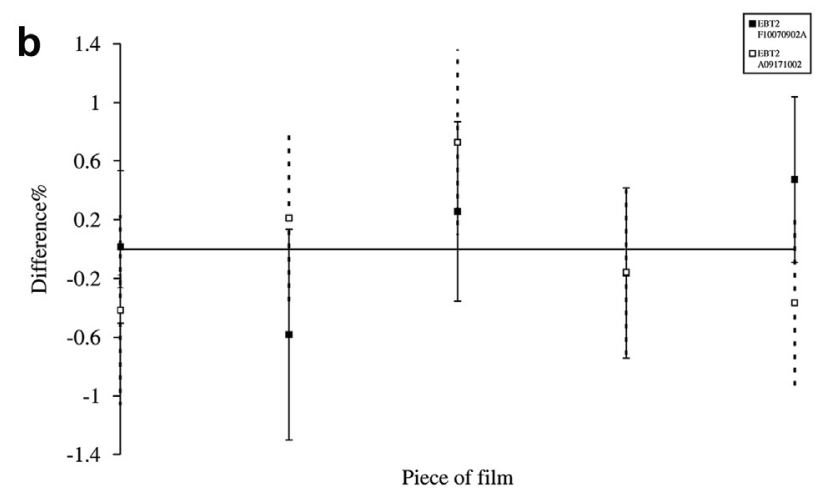

Figure 10 Relative difference of the mean PV resulting from scanning pieces of the same film irradiated with 200 cGy a) EBT b) EBT2 new batch (solid square), old batch (empty square). The error bars correspond to one standard deviation calculated by error propagation from the expression $\left(P V-P V_{\text {mean }}\right)^{*} 100 / P V_{\text {mean }}$. same EBT2 film for the earlier batch (Fig. 10b). Table 1 shows the intrasheet homogeneity results for three dose levels. For the EBT film, the uniformity results were similar, providing a maximum deviation from the mean for $200 \mathrm{cGy}$ in the range of $[-0.7 \%]$ to [0.6\%] equivalent to a dose of $7.0 \pm 2.9 \mathrm{cGy}$. No significant differences between the two batches of EBT2 films were found, nor were there differences between them and the EBT model batch (Fig. $10 \mathrm{a}$ ).

3. Although the results for intersheet and intrasheet homogeneity were very similar in both models, there was a difference in the signal-to-noise ratio (SNR). The noise for the EBT2 was always greater than the EBT; for the old EBT2 batch the maximum relative difference from EBT was $58 \%$ for low doses (20 cGy), and for the new EBT2 batch it was $27 \%$. The SNR for the EBT was greater than the EBT2 (Fig. 11). The SNR result for recent EBT2 batch showed significant improvement. It was reduced for low doses by $37 \%$ for old EBT2 batch and by $21 \%$ for new EBT2 batch compared to SNR for EBT; even the SNR for the new EBT2 batch was greater than the EBT at high doses.

4. Figure 12 shows relative dose profile from EBT2 and EBT model. The dose profile from EBT had the lowest standard deviation (0.9\%); whereas the dose profile from the old and the new batch of the EBT2 film had similar standard deviation (1.5\% and $1.4 \%$ respectively). The Levene test rejects the assumption of equality of variances between EBT dose profile and old EBT2 dose

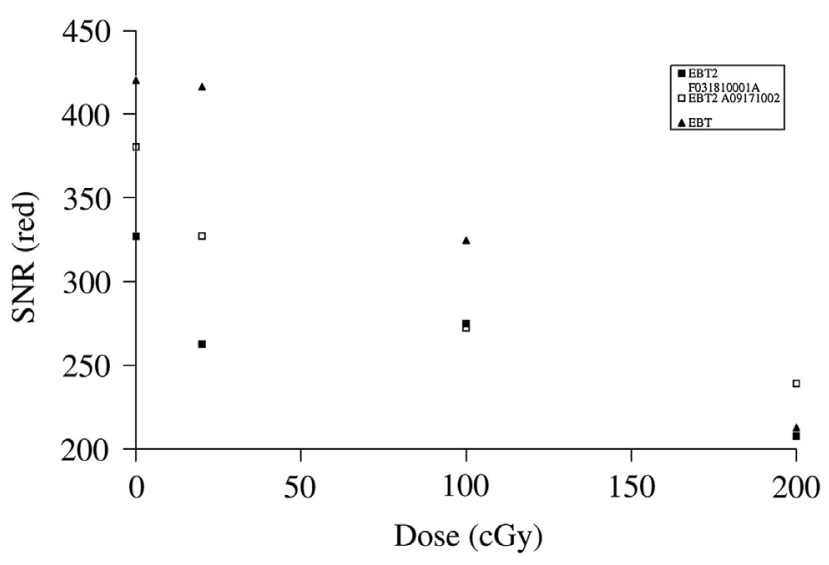

Figure 11 SNR of scanning pieces of the same film irradiated with different doses for both models (the solid squares represent SNR for EBT2 old batch, empty squares represent the new batch model, and solid triangles represent SNR for EBT model). 


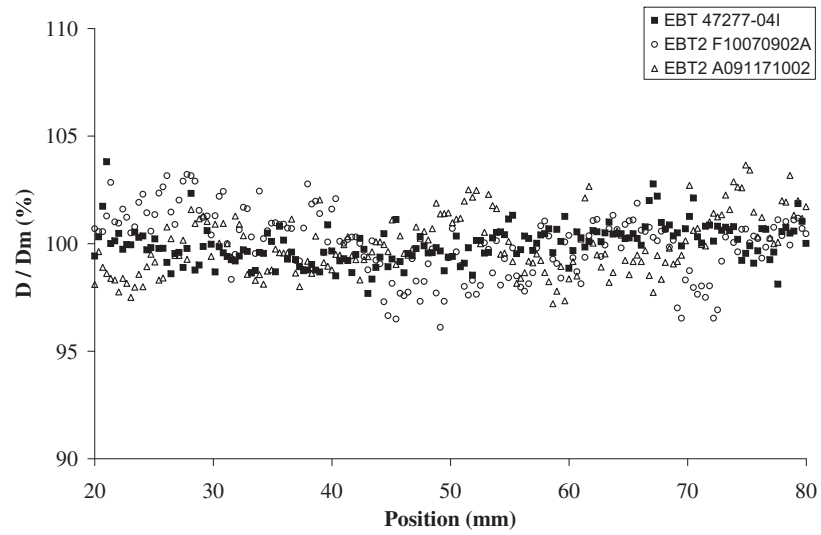

Figure 12 Relative difference from the mean dose $\left(D_{\mathrm{m}}\right)$ for the central points of a horizontal profile for a $10 \times 10$ field: EBT (solid square), new batch EBT2 (empty triangles), old batch EBT2 (empty circles). The uncertainties were around 1-2\% (one standard deviation) and they are not shown for clarity.
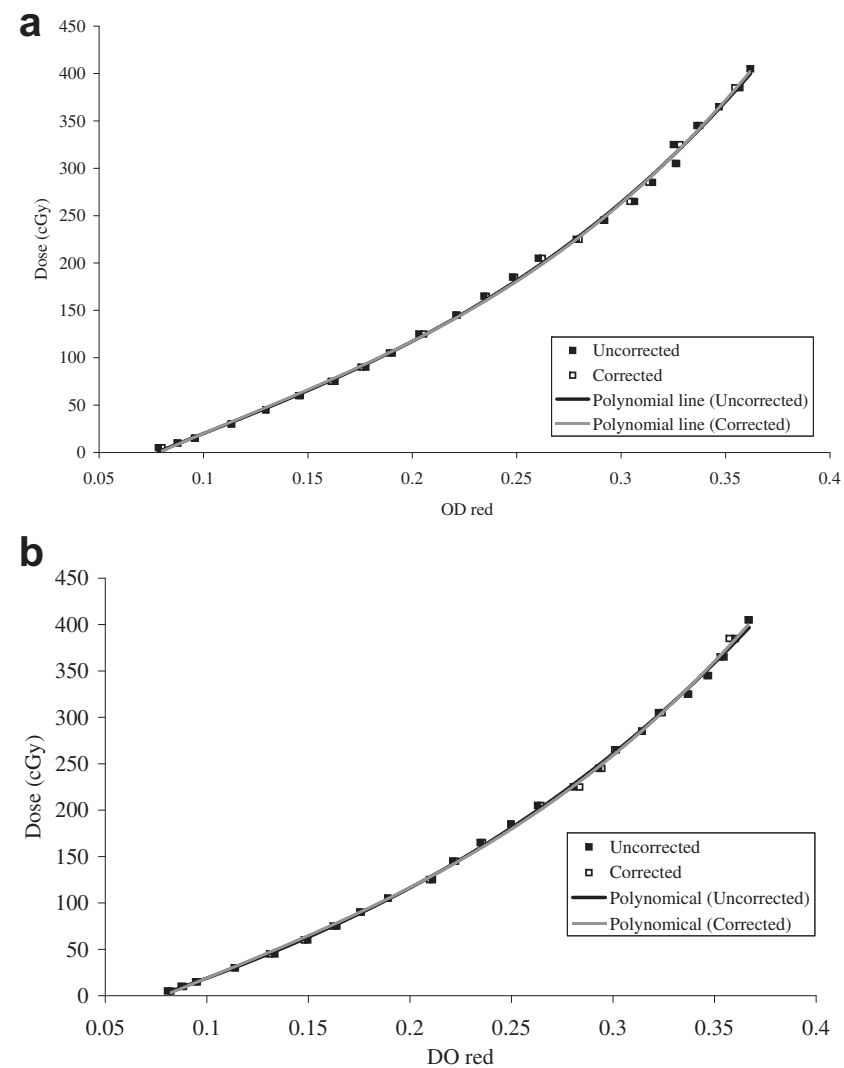

Figure 13 Calibration curves OD-Dose (cGy) for two batches of EBT2 films: a) F031810001A batch b) A09171002 batch. The filled squares represent the uncorrected red OD-dose calibration curve, while the empty squares represent the corrected red OD-Dose calibration curve. The points were fitted to a third order polynomial and the linear correlation coefficient values $R^{2}$ were obtained (F031810001A: uncorrected $R^{2}=0.9980$, corrected $R^{2}=0.9991$; A09171002: uncorrected $R^{2}=0.9992$, corrected $\left.R^{2}=0.9990\right)$. The uncertainties were lower than 0.003 (one standard deviation) and they are not shown for clarity.

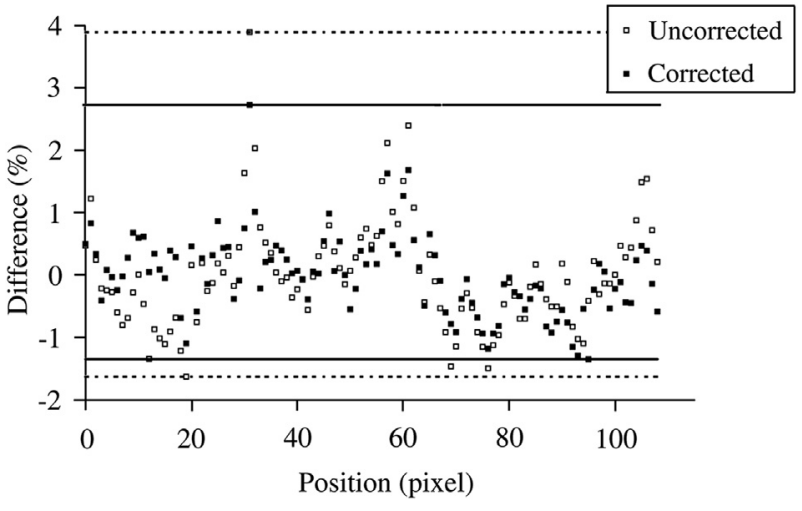

Figure 14 Differences from the mean OD (\%) for the points of a horizontal profile for a piece of film irradiated with $100 \mathrm{cGy}$. The solid squares represent the values of $O D$ for the red channel corrected and the empty squares are the OD values uncorrected. The discontinuous line represents the maximum and minimum difference for the uncorrected OD red, and the continuous line for the corrected OD red. The uncertainties were around $1 \%$ (one standard deviation) and they are not shown for clarity.

profile $(p=0.000)$ and between EBT dose profile and new EBT2 dose profile ( $p=0.003)$, however there were no significant differences between EBT2 batches $(p=0.466)$.

5. Figure 13 shows calibration curves with and without manufacturer correction for both EBT2 batches. The fit function was very similar before and after correction for both batches. This fact is the reason why the manufacturer recommends [42], in order to be practical, to not correct the calibration films and just use the calibration data before correction.

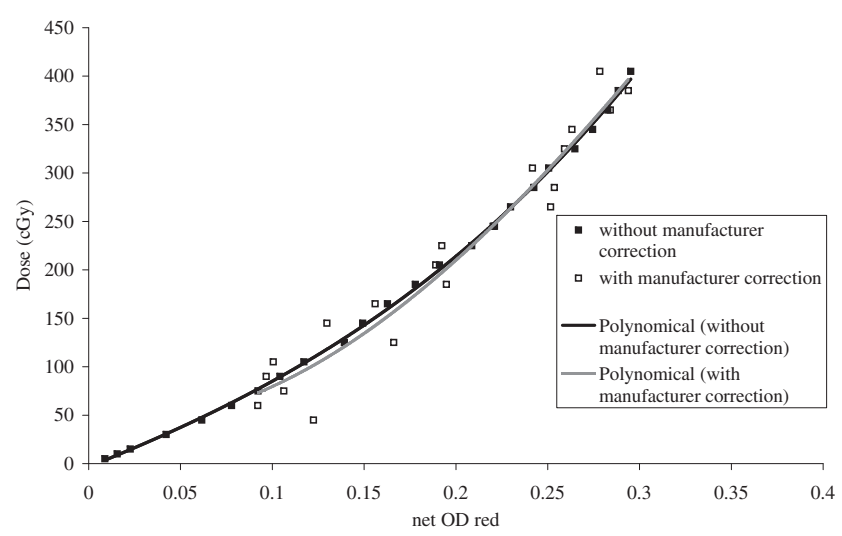

Figure 15 Calibration curves net OD-Dose (cGy) for EBT2 $A 09117002$. The filled squares represent the uncorrected red net OD-dose calibration curve, while the empty squares represent the corrected red net OD-Dose calibration curve. The points were fitted to a third order polynomial and the linear correlation coefficient values $R^{2}$ were obtained (uncorrected $R^{2}=0.9992$, corrected $R^{2}=0.9471$ ). The uncertainties were lower than 0.003 (one standard deviation) and they are not shown for clarity. 
The application of the correction method proposed by the manufacturer to the profiles showed a decrease in the inhomogeneity of corrected $O D_{\text {red }}$ values against the uncorrected $\mathrm{OD}_{\text {red }}$ values. Figure 14 represents the corrected and uncorrected $O D$ for the case in which the standard deviation of the profile diminishes more: $100 \mathrm{cGy}$ and F031810001A batch. The corrected profile was more uniform than the uncorrected profile. In all analyzed profiles, the standard deviation of both optical density and dose decreased when we applied the correction. Nevertheless, we only found statistical significance for moderate doses (100 cGy) in the old EBT2 batch ( $p=0.03)$. For high doses (200 cGy) we found no statistical significance in any batch analyzed; this result is in good agreement with that specified by the manufacturer [41]: marker dye correction is needed at lower dose, whereas the scanner noise dominates at higher doses.

On the other hand, when we used net optical density instead of optical density for the calibration curve, the fit function was highly equivocal after correction (Fig. 15). This result agrees with that observed by Kairn [29]. The study realized by Kairn [29] on the use of the manufacturer's correction showed an increase in the level of the noise of the profiles when the correction is applied. Nevertheless, Kairn used the net optical density instead of optical density.

Finally, when we applied pre-irradiated pixel value correction to the profiles we only found statistical significance $(p=0.004)$ for the new batch and moderate dose (100 cGy).

\section{Conclusions}

We investigated the influence that different parameters have on the EBT2 film dosimetry, especially those modified from the EBT. EBT2 film dependence on light exposure is lower than EBT; differences in pixel values remained below $0.5 \%$ for a minimum of 15 days. On the other hand, the asymmetry of EBT2 configuration implies a new dependence on the 'front-back' film orientation; the pixel value difference between them could reach up to $1 \%$. The most controversial issue is film homogeneity. We studied the EBT2 homogeneity among sheets of the same batch, and the homogeneity of pieces of a single sheet; in both cases the inhomogeneity is less than $1 \%$, in relative pixel value from the mean. This value is similar to the EBT inhomogeneity. However, we found that the SNR was reduced for low doses by $37 \%$ for old EBT2 batch and by $21 \%$ for new EBT2 batch compared to signal-to-noise ratio for EBT. The dose profile from EBT had lower standard deviation $(0.9 \%)$ than the dose profiles from both EBT2 batches (1.5\% and $1.4 \%$ for the old and the new batch respectively); there were significant standard deviation differences between EBT and EBT2 ( $p=0.000$ (old), $p=0.003$ (new)), although the differences between EBT2 batches were no significant. We tested the homogeneity correction protocol proposed by the manufacturer to take into account the film's response deviations by small differences in the active layer's thickness. When we applied the proposed correction, the standard deviation of the dose distribution profile decreased slightly in the two batches studied. Similarly, the standard deviation diminished when we applied the pre-irradiated pixel value correction method. However, none of the analyzed correction methods showed clear statistical significance. Our results did not show a clear advantage between using net optical density without correction and using optical density with correction. These results are valid for the batches studied but the process of making EBT2 films is stabilizing and should verify that subsequent batches meet the same characteristics, especially with regard to homogeneity.

\section{References}

[1] Fiandra C, Ricardi U, Ragona R, Anglesio S, Giglioli F, Calamia E, et al. Clinical use of EBT model Gafchromic film in radiotherapy. Med Phys 2006;33:4314-9.

[2] Chiu-Tsau ST, Chan MF. Photon beam dosimetry in the superficial buildup region using radiochromic EBT film stack. Med Phys 2009;36:2074-83.

[3] Devic S, Seuntjens J, Abdel-Rahman W, Evans M, Olivares M, Podgorsak EB, et al. Accurate skin dose measurements using radiochromic film in clinical applications. Med Phys 2006;33: 1116-24.

[4] Zeidan O, Stephenson S, Meeks S, Wagner T, Willoughby T, Kupelian $\mathrm{P}$, et al. Characterization and use of EBT radiochromic film for IMRT dose verification. Med Phys 2006;33:4064-72.

[5] Wilcox E, Daskalov G, Nedialkova L. Comparison of the epson expression 1680 flatbed and the vidar VXR-16 dosimetry PRO film scanners for use in IMRT dosimetry using Gafchromic and radiographic film. Med Phys 2007;34:41-8.

[6] Trichter S, Trichter F, Sabbas A, Lovelock D, Kulidzhanov F, Nori D. Evaluation of Gafchromic EBT film for IMRT QA using two different scanners. Med Phys 2005;32:2167.

[7] Schneider F, Polednik M, Wolff D, Steil W, Delana A, Wenz F, et al. Optimization of the Gafchromic EBT protocol for IMRT QA. Z Med Phys 2009;19:29-37.

[8] Chiu-Tsao D, Medich D, Munro J. The use of new GAFCHROMIC EBT film for $125 \mathrm{I}$ seed dosimetry in solid water phantom. Med Phys 2008;35:3787-99.

[9] Sarfehnia A, Kawrakow I, Seuntjens J. Direct measurement of absorbed dose to water in HDR Ir-192 brachytherapy: water calorimetry, ionization chamber, Gafchromic film, and TG-43. Med Phys 2010;37:1924-32.

[10] Lorenz F, Nalichowski A, Rosca F, Kung J, Wenz F, Zygmanski P. Spatial dependence of MLC transmission in IMRT delivery. Phys Med Biol 2007;52:5985-99.

[11] Fontanarosa D, Orlandini L, Andriani I, Bernardi L. Commissioning varian enhanced dynamic wedge in the PINNACLE treatment planning system using Gafchromic EBT film. Med Phys 2009;36:4504-10.

[12] International Specialty Products. Gafchromic ${ }^{\circledR}$ EBT selfdeveloping film for radiotherapy dosimetry. Available from: http://online1.ispcorp.com/_layouts/Gafchromic/content/ products/ebt/pdfs/EBTwhitepaper.pdf; 2007.

[13] Devic S, Seuntjens J, Sham E, Podgorask E, Ross C, Kirov A, et al. Precise radiochromic film dosimetry using a flat-bed document scanner. Med Phys 2005;32:2245-53.

[14] Martisikova M, Ackermann B, Jäkel O. Analysis of uncertainties in Gafchromic EBT film dosimetry of photons beams. Phys Med Biol 2008;53:7013-27.

[15] Fuss M, Sturtewagen E, De Wagter C, Georg D. Dosimetric characterization of Gafchromic EBT film and its implication on film dosimetry quality assurance. Phys Med Biol 2007;52:4211-25.

[16] Ferreira B, Lopes M, Capela M. Evaluation of an epson flatbed scanner to read Gafchromic EBT films for radiation dosimetry. Phys Med Biol 2009;54:1073-85. 
[17] Niroomand-Rad A, Blackwell C, Coursey B, Gall K, Galvin J, McLaughlin $\mathrm{W}$, et al. Radiochromic film dosimetry recommendations of AAPM radiation therapy committee task group. Med Phys 1998;25:2093-115.

[18] International Speciality Products. Gafchromic ${ }^{\circledR}$ EBT2 selfdeveloping film for radiotherapy dosimetry. Available from: http://online1.ispcorp.com/_layouts/Gafchromic/content/ products/ebt/pdfs/GAFCHROMICEBT2TechnicalBrief-Rev 1.pdf.

[19] Arjomandy B, Taylor R, Anand A, Sahoo N, Gillin M, Prado K, et al. Energy dependence and dose response of Gafchromic EBT2 film over a wide range of photon, electron, and proton beam energies. Med Phys 2010;37:1942-7.

[20] Butson MJ, Yu PK, Cheung T, Alnawaf H. Energy response of the new EBT2 radiochromic film to $x$-ray radiation. Rad Meas 2010;45:836-9.

[21] Lindsay P, Rink A, Ruschin M, Jaffray D. Investigation of energy dependence of EBT and EBT-2 Gafchromic film. Med Phys 2010;37:571-6.

[22] Sutherland JGH, Rogers DWO. Monte Carlo calculated absorbed-dose energy dependence of EBT and EBT2 film. Med Phys 2010;37:1110-6.

[23] Aldelaijan S, Devic S, Mohammed H, Tomic N, Liang L, DeBlois F. Evaluation of EBT-2 model GAFCHROMIC ${ }^{\text {TM }}$ film performance in water. Med Phys 2010;37:3687-93.

[24] Butson MJ, Cheung T, Yu PK. Dose and absorption spectra response of EBT2 Gafchromic film to high energy x-rays. Australas Phys Eng Sci Med 2009;32:196-202.

[25] Desroches J, Bouchard H, Lacroix F. Potencial errors in optical density measurements due to scanning side in EBT and EBT2 Gafchromic film dosimetry. Med Phys 2010;37:1565-70.

[26] Devic S, Aldelaijan S, Mohammed H, Tomic N, Liang LH, DeBlois F. Absorption spectra time evolution of EBT-2 model GAFCHROMIC ${ }^{\text {TM }}$ film. Med Phys 2010;37:2207-14.

[27] Richley L, John AC, Coomber H, Fletcher S. Evaluation and optimization of the new EBT2 radiochromic film dosimetry system for patient dose verification in radiotherapy. Phys Med Biol 2010;55:2601-17.

[28] Hartmann B, Martisíková $M$, Jäkel O. Homogeneity of Gafchromic $^{\circledR}$ EBT2 film. Med Phys 2010;37:1753-6.
[29] Kairn T, Aland T, Kenny J. Local heterogeneities in early batches of EBT2 film: a suggested solution. Phys Med Biol 2010;55:L37-42.

[30] Devic S. Radiochromic film dosimetry: past, present, and future. Physica Med 2011;27:122-34.

[31] Andrés C, Del Castillo A, Tortosa R, Alonso D. A comprehensive study of the Gafchromic EBT2 radiochromic film. A comparison with EBT. Med Phys 2010;37:6271-8.

[32] ImageJ software. Available from: http://rsbweb.nih.gov/ij/.

[33] Devic S, Tomic N, Pang Z, Seuntjens J, Podgorsak EB, Soares CG. Absorption spectroscopy of EBT model GAFCHROMIC $^{\text {TM }}$ film. Med Phys 2007;34:112-8.

[34] Paelinck L, De Neve W, De Wagter C. Precautions and strategies in using commercial flatbed scanner for radiochromic film dosimetry. Phys Med Biol 2007;52:231-42.

[35] Devic S, Seuntjens J, Hegyi G, Podgorsak E, Soares C, Kirov A, et al. Dosimetric properties of improved Gafchromic films for seven different digitizers. Med Phys 2004;31:2392-401.

[36] Lynch B, Kozelka J, Ranade M, Li J, Simon W, Dempsey JF. Important considerations for radiochromic film dosimetry with flatbed CCD scanners and EBT GAFCHROMIC film. Med Phys 2006;33:4551-6.

[37] Cheung T, Butson M, Yu P. Post-irradiation coloration of Gafchromic EBT radiochromic film. Phys Med Biol 2005;50:281-5.

[38] Devic S, Wang YZ, Tomic N, Podgorsak EB. Sensitivity of linear CCD array based film scanners used for film dosimetry. Med Phys 2006;33:3993-6.

[39] Miras H, Arrans R. An easy method to account for light scattering dose dependence in radiochromic films. Med Phys 2009; 36:3866-70.

[40] Menegotti L, Delano A, Martignano A. Radiochromic film dosimetry with flatbed scanners: a fast and accurate method for dose calibration and uniformity correction with single film exposure. Med Phys 2008;35:3078-86.

[41] Micke A, Lewis D, Yu X. International Specialty Products. Tripple Channel Technique Film Dosimetry. Available from: http://www.gafchromic.com/.

[42] GafchromicEBT2CorrectionProtocol-Rev1.pdf. Available from: http://www.gafchromic.com/. 